(C) 2003 International Press

Adv. Theor. Math. Phys. 7 (2003) 25-52

\title{
The Long-Time Dynamics of Dirac Particles in the Kerr-Newman Black Hole Geometry
}

\author{
Felix Finster \\ NWF I - Mathematik \\ Universität Regensburg, Germany \\ Felix.Finster@mathematik.uni-regensburg.de \\ Niky Kamran \\ Department of Math. and Statistics \\ McGill University, Montréal, Canada \\ nkamran@math.McGill.CA \\ Joel Smoller \\ Mathematics Department \\ The University of Michigan, Ann Arbor, MI \\ smoller@umich.edu \\ Shing-Tung Yau \\ Mathematics Department \\ Harvard University, Cambridge, MA \\ yau@math.harvard.edu
}

\begin{abstract}
We consider the Cauchy problem for the massive Dirac equation in the non-extreme Kerr-Newman geometry outside the event horizon. We derive an integral representation for the Dirac propagator involving the solutions of the ODEs which arise in Chandrasekhar's separation of variables. It is proved that for initial data in $L_{\mathrm{loc}}^{\infty}$ near the event horizon with $L^{2}$ decay at infinity, the probability of the Dirac particle to be in any compact region of space tends to zero as $t$ goes to infinity. This means that the Dirac particle must either disappear in the black hole or escape to infinity.
\end{abstract}

e-print archive: http://lanl.arXiv.org/abs/http://xxx.lanl.gov/abs/gr-qc/0005088 


\section{Introduction}

It has recently been shown that the Dirac equation does not admit normalizable, time-periodic solutions in the non-extreme Kerr-Newman axisymmetric black hole geometry [1]. This was interpreted as an indication that a Dirac particle either falls into the black hole or escapes to infinity, but that it cannot stay in a bounded region outside the event horizon. In this paper we make precise this interpretation in the general time-dependent setting. We thus consider the Cauchy problem for the Dirac equation with smooth initial data on the hypersurface $t=0$, compactly supported outside the event horizon. We study the probability for the Dirac particle to be inside a given annulus located outside the event horizon, and we prove that this probability tends to zero as $t$ goes to infinity. Hence, in contrast to the situation for a bounded orbit of a classical point particle, there exists no compact region outside the event horizon in which the quantum mechanical Dirac particle will remain for all time. In more precise form, our result is stated as follows. Recall that in Boyer-Lindquist coordinates $(t, r, \vartheta, \varphi)$ with $r>0,0 \leq \vartheta \leq \pi$, $0 \leq \varphi<2 \pi$, the Kerr-Newman metric takes the form [2]

$$
\begin{aligned}
d s^{2}= & g_{j k} d x^{j} x^{k} \\
= & \frac{\Delta}{U}\left(d t-a \sin ^{2} \vartheta d \varphi\right)^{2}-U\left(\frac{d r^{2}}{\Delta}+d \vartheta^{2}\right) \\
& -\frac{\sin ^{2} \vartheta}{U}\left(a d t-\left(r^{2}+a^{2}\right) d \varphi\right)^{2}
\end{aligned}
$$

with

$$
U(r, \vartheta)=r^{2}+a^{2} \cos ^{2} \vartheta, \quad \Delta(r)=r^{2}-2 M r+a^{2}+Q^{2},
$$

and the electromagnetic potential is given by

$$
A_{j} d x^{j}=-\frac{Q r}{U}\left(d t-a \sin ^{2} \vartheta d \varphi\right),
$$

where $M, a M$ and $Q$ denote the mass, the angular momentum and the charge of the black hole, respectively. We shall here restrict attention to the non-extreme case $M^{2}>a^{2}+Q^{2}$. Then the function $\Delta$ has two distinct zeros,

$$
r_{0}=M-\sqrt{M^{2}-a^{2}-Q^{2}} \text { and } r_{1}=M+\sqrt{M^{2}-a^{2}-Q^{2}},
$$

corresponding to the Cauchy and the event horizon, respectively. We will here consider only the region $r>r_{1}$ outside of the event horizon, and thus $\Delta>0$.

Theorem 1.1. Consider the Cauchy problem for the Dirac equation in the non-extreme Kerr-Newman black hole geometry outside the event horizon

$$
\left(i \gamma^{j} D_{j}-m\right) \Psi(t, x)=0, \quad \Psi(0, x)=\Psi_{0}(x),
$$


where the initial data $\Psi_{0}$ is in $L^{2}\left(\left(r_{1}, \infty\right) \times S^{2}, d \mu\right)^{4}$, where $d \mu$ is the induced invariant measure on the hypersurface $t=$ const. Then for any $\delta>0$ and $R>r_{1}+\delta$, the probability for the Dirac particle to be inside the annulus $K_{\delta, R}=\left\{r_{1}+\delta \leq r \leq R\right\}$ tends to zero as $t \rightarrow \infty$, i.e.

$$
\lim _{t \rightarrow \infty} \int_{K_{\delta, R}}\left(\bar{\Psi} \gamma^{j} \Psi\right)(t, x) \nu_{j} d \mu=0
$$

where $\nu$ denotes the future directed normal.

The decay of probabilities in compact sets (1.3) can be stated equivalently that the Dirac wave function decays in $L_{\text {loc }}^{2}$ outside and away from the event horizon. Since the Dirac equation is linear and stationary, for smooth initial data we obtain immediately that also the time-derivatives $\partial_{t}^{n} \Psi$ decay in $L_{\mathrm{loc}}^{2}$, and standard Sobolev methods yield that $\Psi$ decays even in $L_{\mathrm{loc}}^{\infty}$. We point out that the initial data is merely bounded (but not necessarily small), near the event horizon. Our assumptions include the case when the initial data is smooth and bounded in the maximal Kruskal extension up to the bifurcation 2-sphere (as is considered in [7] for the wave function in the Schwarzschild geometry). We note that the axisymmetric character of the background geometry makes the analysis significantly more delicate than in the spherically symmetric case, mainly because for $a \neq 0$ both the radial and angular ODEs depend on the energy, and thus for the study of the dynamics we must consider the system of these coupled equations.

The proof is organized as follows. We first bring the Dirac equation into the Hamiltonian form $i \partial_{t} \Psi=H \Psi$ with a self-adjoint operator $H$. Our main technical tool is an integral representation for the Dirac propagator $\exp (-i t H)$ acting on wave functions with compact support outside the event horizon. This integral representation is stated in Theorem 3.6. To derive it, we first consider the Dirac equation in an annulus outside the event horizon with suitable Dirichlet-type boundary conditions. The Hamiltonian corresponding to this modified problem has a purely discrete spectrum, and thus the propagator can be decomposed into discrete eigenstates. We then take the infinite-volume limit by letting the inner boundary of the annulus tend to the event horizon and the outer boundary to infinity in a suitable way. Our construction is based on Chandrasekhar's separation of variables for the Dirac equation in the Kerr-Newman metric $[3,4,5]$ together with estimates for the asymptotic behavior of the amplitudes and phases of the separated radial eigenfunctions (Lemmas 3.1 and 3.5), and for the spectral gaps (Lemma 3.3). The usefulness of our integral representation for the propagator is that it explicitly gives the continuous spectral measure of $H$ in terms of the solutions of the radial and angular ODEs arising in Chandrasekhar's separation 
of variables. For initial data which is compactly supported outside the event horizon, the decay of the probabilities (1.3) then follows by standard results of Fourier analysis. The generalization to initial data in $L^{2}$ and $L_{\text {loc }}^{\infty}$ near the event horizon is done by approximation in our Hilbert space framework.

\section{Separation of Variables, Hamiltonian Formula- tion}

The Dirac equation in the Kerr-Newman geometry can be completely separated into ODEs by Chandrasekhar's method [3, 4, 5]. We here outline the separation, see [1] for details. After the regular and time-independent transformation

$$
\Psi \rightarrow \hat{\Psi}=S \Psi
$$

with

$$
\begin{aligned}
S=\Delta^{\frac{1}{4}} \operatorname{diag}( & (r-i a \cos \vartheta)^{\frac{1}{2}},(r-i a \cos \vartheta)^{\frac{1}{2}}, \\
& \left.(r+i a \cos \vartheta)^{\frac{1}{2}},(r+i a \cos \vartheta)^{\frac{1}{2}}\right),
\end{aligned}
$$

the Dirac equation can be written as

$$
(\mathcal{R}+\mathcal{A}) \hat{\Psi}=0
$$

with

$$
\begin{aligned}
\mathcal{R} & =\left(\begin{array}{cccc}
i m r & 0 & \sqrt{\Delta} \mathcal{D}_{+} & 0 \\
0 & -i m r & 0 & \sqrt{\Delta} \mathcal{D}_{-} \\
\sqrt{\Delta} \mathcal{D}_{-} & 0 & -i m r & 0 \\
0 & \sqrt{\Delta} \mathcal{D}_{+} & 0 & i m r
\end{array}\right) \\
\mathcal{A} & =\left(\begin{array}{cccc}
-a m \cos \vartheta & 0 & 0 & \mathcal{L}_{+} \\
0 & a m \cos \vartheta & -\mathcal{L}_{-} & 0 \\
0 & \mathcal{L}_{+} & -a m \cos \vartheta & 0 \\
-\mathcal{L}_{-} & 0 & 0 & a m \cos \vartheta
\end{array}\right)
\end{aligned}
$$

and the differential operators

$$
\begin{aligned}
& \mathcal{D}_{ \pm}=\frac{\partial}{\partial r} \mp \frac{1}{\Delta}\left[\left(r^{2}+a^{2}\right) \frac{\partial}{\partial t}+a \frac{\partial}{\partial \varphi}-i e Q r\right] \\
& \mathcal{L}_{ \pm}=\frac{\partial}{\partial \vartheta}+\frac{\cot \vartheta}{2} \mp i\left[a \sin \vartheta \frac{\partial}{\partial t}+\frac{1}{\sin \vartheta} \frac{\partial}{\partial \varphi}\right] .
\end{aligned}
$$


Employing the ansatz

$$
\hat{\Psi}(t, r, \vartheta, \varphi)=e^{-i \omega t} e^{-i\left(k+\frac{1}{2}\right) \varphi}\left(\begin{array}{c}
X_{-}(r) Y_{-}(\vartheta) \\
X_{+}(r) Y_{+}(\vartheta) \\
X_{+}(r) Y_{-}(\vartheta) \\
X_{-}(r) Y_{+}(\vartheta)
\end{array}\right), \quad k \in \mathbf{Z}
$$

we obtain the eigenvalue problems,

$$
\mathcal{R} \hat{\Psi}=\lambda \hat{\Psi}, \quad \mathcal{A} \hat{\Psi}=-\lambda \hat{\Psi}
$$

under which the Dirac equation (2.2) decouples into the system of ODEs

$$
\begin{aligned}
\left(\begin{array}{cc}
\sqrt{\Delta} \mathcal{D}_{+} & i m r-\lambda \\
-i m r-\lambda & \sqrt{\Delta} \mathcal{D}_{-}
\end{array}\right)\left(\begin{array}{c}
X_{+} \\
X_{-}
\end{array}\right) & =0 \\
\left(\begin{array}{cc}
\mathcal{L}_{+} & -a m \cos \vartheta+\lambda \\
a m \cos \vartheta+\lambda & -\mathcal{L}_{-}
\end{array}\right)\left(\begin{array}{c}
Y_{+} \\
Y_{-}
\end{array}\right) & =0,
\end{aligned}
$$

where $\mathcal{D}_{ \pm}$and $\mathcal{L}_{ \pm}$are the radial and angular operators

$$
\begin{aligned}
& \mathcal{D}_{ \pm}=\frac{\partial}{\partial r} \pm \frac{i}{\Delta}\left[\omega\left(r^{2}+a^{2}\right)+\left(k+\frac{1}{2}\right) a+e Q r\right] \\
& \mathcal{L}_{ \pm}=\frac{\partial}{\partial \vartheta}+\frac{\cot \vartheta}{2} \mp\left[a \omega \sin \vartheta+\frac{k+\frac{1}{2}}{\sin \vartheta}\right] .
\end{aligned}
$$

We will in what follows also use the vector notation $X=\left(X_{+}, X_{-}\right), Y=$ $\left(Y_{-}, Y_{+}\right)$and for clarity sometimes add indices for the parameters involved, e.g. $X^{k \omega \lambda} \equiv X$. We point out that (2.3) is an eigenfunction of the angular operator $i \partial_{\varphi}$ with eigenvalue $k+\frac{1}{2}$. The reason why we need to consider half odd integer eigenvalues is that the transformation from the usual singlevalued wave function in space-time, to the wave function $\hat{\Psi}$ in $(2.2)$ involves a sign flip at $\varphi=0$ (see $[1$, Section 2.1]).

In this paper, we want to study time-dependent solutions of the Dirac equation. In the separation ansatz (2.3), the dynamics of the solution is encoded through the $\omega$-dependence in the ODEs (2.5) and (2.6). Unfortunately, both the radial and angular operators (2.7) and (2.8) depend on $\omega$, making the situation rather complicated. Therefore it is useful to bring the Dirac equation (2.2) into Hamiltonian form, in a way which is compatible with the separation of variables. We first bring the time derivative in (2.2) to one side of the equation and obtain

$$
\left(\frac{r^{2}+a^{2}}{\sqrt{\Delta}} B+a \sin \vartheta C\right) i \frac{\partial}{\partial t} \hat{\Psi}=\left(\mathcal{R}^{3}+\mathcal{A}^{3}\right) \hat{\Psi}
$$


with

$$
B=\left(\begin{array}{cccc}
0 & 0 & -i & 0 \\
0 & 0 & 0 & i \\
i & 0 & 0 & 0 \\
0 & -i & 0 & 0
\end{array}\right), \quad C=\left(\begin{array}{cccc}
0 & 0 & 0 & -1 \\
0 & 0 & -1 & 0 \\
0 & -1 & 0 & 0 \\
-1 & 0 & 0 & 0
\end{array}\right)
$$

where the operators $\mathcal{R}^{3}$ and $\mathcal{A}^{3}$ are obtained from $\mathcal{R}$ and $\mathcal{A}$ by setting the time derivatives to zero. The matrices $B$ and $C$ satisfy the relations $B^{2}=\mathbb{1}=C^{2}$ and $B C=C B$. Thus the linear combination of these matrices which appears in $(2.9)$ can be inverted with the formula $(\alpha B+\beta C)^{-1}=$ $\left(\alpha^{2}-\beta^{2}\right)^{-1}(\alpha B-\beta C)$ (and $\left.\alpha, \beta \in \mathbb{R}\right)$. Furthermore, we introduce a new radial variable $u \in(-\infty, \infty)$ by

$$
\frac{d u}{d r}=\frac{r^{2}+a^{2}}{\Delta} .
$$

Omitting for simplicity the hat of the wave function, the Dirac equation (2.9) becomes

$$
i \frac{\partial}{\partial t} \Psi=H \Psi
$$

with the Hamiltonian

$$
\begin{aligned}
H & =\left(\frac{\left(r^{2}+a^{2}\right)^{2}}{\Delta}-a^{2} \sin ^{2} \vartheta\right)^{-1}\left(\frac{r^{2}+a^{2}}{\sqrt{\Delta}} B-a \sin \vartheta C\right)\left(\mathcal{R}^{3}+\mathcal{A}^{3}\right) \\
& =\left[\left(1-\frac{a^{2} \Delta \sin ^{2} \vartheta}{\left(r^{2}+a^{2}\right)^{2}}\right)^{-1}\left(\mathbb{1}-\frac{a \sqrt{\Delta} \sin \vartheta}{r^{2}+a^{2}} B C\right)\right](\hat{\mathcal{R}}+\hat{\mathcal{A}}), \quad(2.11)
\end{aligned}
$$

where $r$ is an implicit function of $u$, and

$$
\begin{aligned}
\hat{\mathcal{R}}= & -\frac{m r \sqrt{\Delta}}{r^{2}+a^{2}}\left(\begin{array}{cccc}
0 & 0 & 1 & 0 \\
0 & 0 & 0 & 1 \\
1 & 0 & 0 & 0 \\
0 & 1 & 0 & 0
\end{array}\right)+\left(\begin{array}{cccc}
-\mathcal{E}_{-} & 0 & 0 & 0 \\
0 & \mathcal{E}_{+} & 0 & 0 \\
0 & 0 & \mathcal{E}_{+} & 0 \\
0 & 0 & 0 & -\mathcal{E}_{-}
\end{array}\right) \\
\hat{\mathcal{A}}= & \frac{a m \cos \vartheta \sqrt{\Delta}}{r^{2}+a^{2}}\left(\begin{array}{cccc}
0 & 0 & i & 0 \\
0 & 0 & 0 & i \\
-i & 0 & 0 & 0 \\
0 & -i & 0 & 0
\end{array}\right) \\
& +\left(\begin{array}{cccc}
0 & -\mathcal{M}_{+} & 0 & 0 \\
-\mathcal{M}_{-} & 0 & 0 & 0 \\
0 & 0 & 0 & \mathcal{M}_{+} \\
0 & 0 & \mathcal{M}_{-} & 0
\end{array}\right)
\end{aligned}
$$


with

$$
\begin{aligned}
\mathcal{E}_{ \pm} & =i \frac{\partial}{\partial u} \mp\left(\frac{i a}{r^{2}+a^{2}} \frac{\partial}{\partial \varphi}+\frac{e Q r}{r^{2}+a^{2}}\right) \\
\mathcal{M}_{ \pm} & =\frac{\sqrt{\Delta}}{r^{2}+a^{2}}\left(i \frac{\partial}{\partial \vartheta}+i \frac{\cot \vartheta}{2} \pm \frac{1}{\sin \vartheta} \frac{\partial}{\partial \varphi}\right)
\end{aligned}
$$

The Hamiltonian (2.11) is an operator acting on the wave functions on the hypersurfaces $t=$ const. The simplest scalar product on such a hypersurface is

$$
(\Psi \mid \Phi)=\int_{-\infty}^{\infty} d u \int_{-1}^{1} d \cos \vartheta \int_{0}^{2 \pi} d \varphi \bar{\Psi}(t, u, \vartheta, \varphi) \Phi(t, u, \vartheta, \varphi),
$$

where " $\bar{\Psi} "$ denotes the complex conjugated, transposed spinor. In the spherically symmetric case $a=0$, the Hamiltonian (2.11) is Hermitian (i.e. formally self-adjoint) with respect to this scalar product. However for $a \neq 0$, $H$ is not Hermitian. In order to get around this problem, we introduce a different scalar product as follows. Notice that the operators $\hat{\mathcal{R}}$ and $\hat{\mathcal{A}}$, (2.12),(2.13), are both Hermitian with respect to (2.14). The reason why the Hamiltonian (2.11) is not Hermitian is that, when the taking the adjoint of $H$ using integration by parts, one gets $r$ - and $\vartheta$-derivatives of the square bracket in (2.11). But we can compensate this square bracket by inserting its inverse into the scalar product. Thus we introduce on the four-component spinors the inner product

$$
<\Psi \mid \Phi>_{(t, u, \vartheta, \varphi)}=\bar{\Psi}(t, u, \vartheta, \varphi)\left(\mathbb{1}+\frac{a \sqrt{\Delta} \sin \vartheta}{r^{2}+a^{2}} B C\right) \Phi(t, u, \vartheta, \varphi)
$$

and define the scalar product $<. \mid$. $>$ by

$$
<\Psi\left|\Phi>=\int_{-\infty}^{\infty} d u \int_{-1}^{1} d \cos \vartheta \int_{0}^{2 \pi} d \varphi<\Psi\right| \Phi>_{(t, u, \vartheta, \varphi)} .
$$

Then the Hamiltonian $H$ is Hermitian with respect to (2.16). Let us verify that (2.16) is positive. In the region outside the event horizon under consideration, $r>r_{1}>M$. Also, since we are in the non-extreme case, $M>Q, a$, and as a consequence, $\Delta<r^{2}$. We conclude that

$$
\left|\frac{a \sqrt{\Delta} \sin \vartheta}{r^{2}+a^{2}}\right| \leq \frac{a \sqrt{\Delta}}{r^{2}+a^{2}}<\frac{a}{r} \frac{\sqrt{\Delta}}{r}<1 .
$$

Combining this inequality with the fact that the matrix $B C$ has eigenvalues \pm 1 , we obtain that the bracket in $(2.15)$ is indeed a positive matrix. 
We denote the Hilbert space of wave functions with scalar product (2.16) by $\mathcal{H}$. Then the operator $H,(2.11)$, is essentially self-adjoint on $\mathcal{H}$ with domain of definition

$$
D(H)=C_{0}^{\infty}\left(\mathbb{R} \times S^{2}\right)^{4} .
$$

In Section 3, we shall consider the Dirac operator also with certain Dirichlettype boundary conditions, which we now introduce. First for given $u_{2} \in$ $\mathbb{R}$, we restrict $u$ to the half line $u \in\left(-\infty, u_{2}\right]$ and impose the boundary conditions

$$
\Psi_{1}\left(u_{2}, \vartheta, \varphi\right)=\Psi_{3}\left(u_{2}, \vartheta, \varphi\right) \quad \text { and } \quad \Psi_{2}\left(u_{2}, \vartheta, \varphi\right)=\Psi_{4}\left(u_{2}, \vartheta, \varphi\right) .
$$

Let $\mathcal{H}_{u_{2}}$ be the Hilbert space of square integrable wave functions $\Psi(u, \vartheta, \varphi)$, $u \leq u_{2}$ with the scalar product

$$
<\Psi\left|\Phi>_{u_{2}}:=\int_{-\infty}^{u_{2}} d u \int_{-1}^{1} d \cos \vartheta \int_{0}^{2 \pi} d \varphi<\Psi\right| \Phi>_{(t, u, \vartheta, \varphi)} .
$$

Then the Hamiltonian (2.11) on $\mathcal{H}_{u_{2}}$ with boundary conditions (2.17), which we denote for clarity by $H_{u_{2}}$, is Hermitian (the main point here is that the boundary values at $u=u_{2}$ vanish when the adjoint of $H_{u_{2}}$ is calculated using integration by parts). The operator $H_{u_{2}}$ is essentially self-adjoint on $\mathcal{H}_{u_{2}}$ with domain of definition

$$
D\left(H_{u_{2}}\right)=\left\{\Psi \in C_{0}^{\infty}\left(\left(-\infty, u_{2}\right] \times S^{2}\right)^{4} \text { and }(2.17) \text { is satisfied }\right\} .
$$

Similarly, for $u_{1}, u_{2} \in \mathbb{R}, u_{1}<u_{2}$, we restrict $u$ to the closed interval $u \in\left[u_{1}, u_{2}\right]$ with boundary conditions

$$
\begin{array}{ll}
\Psi_{1}\left(u_{1}\right)=\Psi_{3}\left(u_{1}\right), & \Psi_{2}\left(u_{1}\right)=\Psi_{4}\left(u_{1}\right) \\
\Psi_{1}\left(u_{2}\right)=\Psi_{3}\left(u_{2}\right), & \Psi_{2}\left(u_{2}\right)=\Psi_{4}\left(u_{2}\right) .
\end{array}
$$

We denote the Hilbert space of square integrable wave functions $\Psi(u, \vartheta, \varphi)$, $u_{1} \leq u \leq u_{2}$, with the scalar product

$$
<\Psi, \Phi>_{u_{1}, u_{2}}:=\int_{u_{1}}^{u_{2}} d u \int_{-1}^{1} d \cos \vartheta \int_{0}^{2 \pi} d \varphi<\Psi \mid \Phi>_{(t, u, \vartheta, \varphi)}
$$

by $\mathcal{H}_{u_{1}, u_{2}}$, and the Hamiltonian $(2.11)$ on $\mathcal{H}_{u_{1}, u_{2}}$ by $H_{u_{1}, u_{2}}$. It is essentially self-adjoint with

$$
D\left(H_{u_{1}, u_{2}}\right)=\left\{\Psi \in C_{0}^{\infty}\left(\left[u_{1}, u_{2}\right] \times S^{2}\right)^{4} \text { and }(2.19) \text { is satisfied }\right\} .
$$

Our above Hamiltonian formulation of the Dirac equation is well-suited to Chandrasekhar's separation of variables. Namely, the boundary conditions (2.17) reduce to simple boundary conditions for the radial functions,

$$
X_{+}\left(u_{2}\right)=X_{-}\left(u_{2}\right) \text {, }
$$


whereas (2.19) amounts to

$$
X_{+}\left(u_{1}\right)=X_{-}\left(u_{1}\right) \quad \text { and } \quad X_{+}\left(u_{2}\right)=X_{-}\left(u_{2}\right) \text {. }
$$

The scalar product (2.14) splits into the product of a radial and an angular part, namely

$$
\left(\hat{\Psi}^{k \omega \lambda} \mid \hat{\Psi}^{k^{\prime} \omega^{\prime} \lambda^{\prime}}\right)=\left(X^{k \omega \lambda} \mid X^{k^{\prime} \omega^{\prime} \lambda^{\prime}}\right)\left(Y^{k \omega \lambda} \mid Y^{k^{\prime} \omega^{\prime} \lambda^{\prime}}\right)
$$

with

$$
\begin{aligned}
\left(X^{k \omega \lambda} \mid X^{k^{\prime} \omega^{\prime} \lambda^{\prime}}\right) & =\int_{-\infty}^{\infty} \overline{X^{k \omega \lambda}}(u) X^{k^{\prime} \omega^{\prime} \lambda^{\prime}}(u) d u \\
\left(Y^{k \omega \lambda} \mid Y^{k^{\prime} \omega^{\prime} \lambda^{\prime}}\right) & =2 \pi \delta^{k k^{\prime}} \int_{-1}^{1} \overline{Y^{k \omega \lambda}}(\vartheta) Y^{k^{\prime} \omega^{\prime} \lambda^{\prime}}(\vartheta) d \cos \vartheta
\end{aligned}
$$

The scalar product (2.16), however, does not split into a product, more precisely

$$
\begin{aligned}
& <\Psi \mid \Phi>=\left(X^{k \omega \lambda} \mid X^{k^{\prime} \omega^{\prime} \lambda^{\prime}}\right)\left(Y^{k \omega \lambda} \mid Y^{k^{\prime} \omega^{\prime} \lambda^{\prime}}\right) \\
& \quad+a\left(X^{k \omega \lambda}\left|\frac{\sqrt{\Delta}}{r^{2}+a^{2}} \sigma^{2}\right| X^{k^{\prime} \omega^{\prime} \lambda^{\prime}}\right)\left(Y^{k \omega \lambda}\left|\sin \vartheta \sigma^{1}\right| Y^{k^{\prime} \omega^{\prime} \lambda^{\prime}}\right),
\end{aligned}
$$

where $\sigma^{i}$ are the Pauli matrices. This mixing of the radial and angular parts in the scalar product can be understood from the fact that the Kerr-Newman solution is only axisymmetric.

\section{An Integral Representation for the Propagator}

The propagator $\exp (-i t H)$ has the spectral decomposition

$$
e^{-i t H}=\int_{-\infty}^{\infty} e^{-i \omega t} d E_{\omega}
$$

where $d E_{\omega}$ is the spectral measure of $H$. In this section, we shall bring this formula into a more explicit form. This will be done by expressing the spectral measure in terms of solutions of the radial and angular ODEs of the previous section. Since the spectrum of $H$ is continuous, it is not obvious how to relate the spectral measure to the solutions of our ODEs. To bypass this problem, we begin with the spectral decomposition of the operator $H_{u_{1}, u_{2}}$ (which has a purely discrete spectrum), and then deduce the desired integral representation for $\exp (-i t H)$ by taking suitable limits $u_{1} \rightarrow-\infty$ and $u_{2} \rightarrow \infty$. 
As an elliptic operator on a bounded domain, the Hamiltonian $H_{u_{1}, u_{2}}$ has a purely discrete spectrum with finite-dimensional eigenspaces (see [6]). In view of our separation of variables, the most convenient eigenvector basis is the following. First we can choose the basis vectors as eigenvectors of the operator $i \partial_{\varphi}$ with eigenvalue $k+\frac{1}{2}, k \in \mathbf{Z}$. We denote this eigenspace of $i \partial_{\varphi}$ by $\mathcal{H}_{u_{1}, u_{2}}^{k}$, and the restriction of $H_{u_{1}, u_{2}}$ to $\mathcal{H}_{u_{1}, u_{2}}^{k}$ by $H_{u_{1}, u_{2}}^{k}$. Furthermore, the basis vectors can be chosen as eigenvectors of the angular operator $\mathcal{A}$. As is shown in the Appendix, the spectrum of $\mathcal{A}$ on $\mathcal{H}_{u_{1}, u_{2}}^{k}$ is discrete, nondegenerate, and depends smoothly on $\omega$. Thus the eigenvalues of $\mathcal{A}$ can be written as $\lambda_{n}(\omega), n \in \mathbf{Z}$, with $\lambda_{n}<\lambda_{n+1}$ and $\lambda_{n}(.) \in C^{\infty}(\mathbb{R})$. For any given $k \in \mathbf{Z}, \omega \in \sigma\left(H_{u_{1}, u_{2}}^{k}\right)$, and $n \in \mathbf{Z}$, the radial ODE (2.5) has at most one solution satisfying the boundary conditions (2.19). Hence we have for any $k$, $\omega$, and $n$ at most one eigenstate of $H_{u_{1}, u_{2}}$, which we denote by $\Psi_{u_{1}, u_{2}}^{k \omega n}$. The set of $n$ for which such an eigenvector exists is denoted by $N(k, \omega)$. Thus our eigenvector basis is

$$
\left(\Psi_{u_{1}, u_{2}}^{k \omega n}\right)_{k \in \mathbf{Z}, \omega \in \sigma\left(H_{u_{1}, u_{2}}^{k}\right), n \in N(k, \omega)} .
$$

We normalize these eigenfunctions with respect to the scalar product (2.14); more precisely, we normalize both the radial and angular parts according to

$$
\left(X_{u_{1}, u_{2}}^{k \omega n} \mid X_{u_{1}, u_{2}}^{k \omega n}\right)=1, \quad\left(Y^{k \omega n} \mid Y^{k \omega n}\right)=1
$$

with $X$ and $Y$ as in (2.3). Since the angular operator $\mathcal{A}$ is self-adjoint with respect to the scalar product (.|.), its eigenvectors are orthogonal, and thus the eigenfunctions for fixed $k$ and $\omega$ are even orthonormal,

$$
\left(\Psi_{u_{1}, u_{2}}^{k \omega n} \mid \Psi_{u_{1}, u_{2}}^{k \omega n^{\prime}}\right)=\delta^{n n^{\prime}}, \quad n, n^{\prime} \in N(k, \omega) .
$$

We mention for clarity that for different values of $\omega$, the eigenfunctions are in general not orthogonal with respect to (.|.), but since $H_{u_{1}, u_{2}}$ is self-adjoint with respect to $<. \mid .>$, its eigenspaces are orthogonal with respect to the latter scalar product, and thus

$$
<\Psi_{u_{1}, u_{2}}^{k \omega n} \mid \Psi_{u_{1}, u_{2}}^{k^{\prime} \omega^{\prime} n^{\prime}}>=0 \quad \text { for } \omega \neq \omega^{\prime} .
$$

These subtle differences between the two scalar products clearly become irrelevant in the spherically symmetric case $a=0$.

In the basis (3.2), the spectral decomposition (3.1) for $H_{u_{1}, u_{2}}$ can be written as

$$
\begin{aligned}
& e^{-i t H_{u_{1}, u_{2}}} \Psi \\
& \quad=\sum_{k \in \mathbf{Z}} \sum_{\omega \in \sigma\left(H_{u_{1}, u_{2}}^{k}\right)} e^{-i \omega t}\left(\sum_{n, n^{\prime} \in N(k, \omega)} c_{n n^{\prime}} \Psi_{u_{1}, u_{2}}^{k \omega n}<\Psi_{u_{1}, u_{2}}^{k \omega n^{\prime}} \mid \Psi>\right)
\end{aligned}
$$


Here the coefficients $c_{n n^{\prime}}$ must be chosen such that the bracket in (3.5) is the projection of $\Psi$ onto the eigenspace of $H_{u_{1}, u_{2}}^{k}$ corresponding to the eigenvalue $\omega$; more precisely,

$$
c_{n n^{\prime}}=\left(A^{-1}\right)_{n n^{\prime}} \quad \text { with } \quad A_{n n^{\prime}}=<\Psi_{u_{1}, u_{2}}^{k \omega n} \mid \Psi_{u_{1}, u_{2}}^{k \omega n^{\prime}}>.
$$

Notice that the first two sums in (3.5) give a decomposition of $\Psi$ into the orthogonal eigenstates of the operators $i \partial_{\varphi}$ and $H$, respectively, and thus converge in norm in $\mathcal{H}_{u_{1}, u_{2}}$. The bracket in (3.5) is the basis representation of the projector on the respective eigenspace.

Our first goal is to take the limit $u_{1} \rightarrow-\infty$ in (3.5). We expect that in this infinite volume limit, the "energy gaps" $\Delta \omega_{k n}$ between neighboring eigenvalues, defined by

$$
\begin{aligned}
\Delta \omega_{k n}= & \min \left\{\tilde{\omega}_{k n}-\omega_{k n} \mid \tilde{\omega}_{k n}>\omega_{k n}\right\} \quad \text { with } \\
& \omega_{k n}, \tilde{\omega}_{k n} \in \sigma\left(H_{u_{1}, u_{2}}^{k}\right) \text { and } N\left(k, \omega_{k n}\right), N\left(k, \tilde{\omega}_{k n}\right) \neq 0,
\end{aligned}
$$

should tend to zero. The basic idea is to rewrite the sum over the spectrum in (3.5) as Riemann sums which converge to integrals as $u_{1} \rightarrow-\infty$, yielding a formula for the propagator of the Hamiltonian $H_{u_{2}}$. For making this idea mathematically precise, it is essential to get good estimates for $\Delta \omega_{k n}$ and to relate the eigenvectors $\Psi_{u_{1}, u_{2}}^{k \omega n}$ in (3.5) to solutions

$$
\Psi_{u_{2}}^{k \omega n}(u) \quad \text { with } k \in \mathbf{Z}, \omega \in \mathbb{R}, n \in \mathbf{Z}, u \in\left(-\infty, u_{2}\right]
$$

of the Dirac equation with boundary conditions (2.17). We note that the convergence of the series in $n$ is not a real issue. Indeed, using an $L^{2}$ approximation argument, we will show in the proof of Theorem 3.6 that we may restrict attention to a finite number of angular momentum modes.

We denote the radial and angular functions corresponding to $\Psi_{u_{2}}^{k \omega n}$ by $X_{u_{2}}^{k \omega n}$ and $Y^{k \omega n}$, respectively. In the variable $u,(2.10)$, the radial equation (2.5) becomes

$$
\left[\frac{d}{d u}+i \Omega(u)\left(\begin{array}{cc}
1 & 0 \\
0 & -1
\end{array}\right)\right] X=\frac{\sqrt{\Delta}}{r^{2}+a^{2}}\left(\begin{array}{cc}
0 & i m r-\lambda \\
-i m r-\lambda & 0
\end{array}\right) X
$$

with

$$
\Omega(u)=\omega+\frac{\left(k+\frac{1}{2}\right) a+e Q r}{r^{2}+a^{2}},
$$

and where for ease in notation the indices of $X$ were omitted. The next lemma describes the asymptotic behavior of $X(u)$ as $u \rightarrow-\infty$. 
Lemma 3.1. Every nontrivial solution $X$ of (3.7) with boundary conditions (2.21) is asymptotically as $u \rightarrow-\infty$ of the form

$$
X(u)=\left(\begin{array}{cc}
e^{-i \Omega_{0} u} f_{0}^{+} \\
e^{i \Omega_{0} u} f_{0}^{-}
\end{array}\right)+R_{0}(u)
$$

with

$$
\begin{aligned}
f_{0} & \neq 0 \\
\Omega_{0} & =\omega+\frac{\left(k+\frac{1}{2}\right) a+e Q r_{1}}{r_{1}^{2}+a^{2}} \\
\left|R_{0}\right| & \leq c e^{d u}
\end{aligned}
$$

and suitable constants $c, d>0$, which can be chosen locally uniformly in $\omega$.

Proof. Substituting into (3.7) the ansatz

$$
X(u)=\left(\begin{array}{c}
e^{-i \Omega_{0} u} f^{+}(u) \\
e^{i \Omega_{0} u} f^{-}(u)
\end{array}\right),
$$

we obtain for $f$ the equation

$$
\begin{aligned}
\frac{d}{d u} f= & {\left[i\left(\Omega_{0}-\Omega(u)\right)\left(\begin{array}{cc}
1 & 0 \\
0 & -1
\end{array}\right)\right.} \\
& \left.+\frac{\sqrt{\Delta}}{r^{2}+a^{2}}\left(\begin{array}{cc}
0 & e^{2 i \Omega u}(-i m r-\lambda)
\end{array}\right)\right] f
\end{aligned}
$$

The square bracket vanishes on the event horizon $r=r_{1}$. In the variable $u$, this leads to exponential decay for $u \rightarrow-\infty$, in the sense that there are constants $c_{1}, d>0$ such that

$$
\left|\frac{d}{d u} f\right| \leq c_{1} e^{d u}|f|
$$

Since $X$ is a nontrivial solution, $|f| \neq 0$. Thus we can divide (3.14) by $|f|$ and integrate from any $u<u_{2}$ to $u_{2}$ to obtain

$$
\left.\log |f|\right|_{u} ^{u_{2}} \leq\left. c_{2} e^{d u}\right|_{u} ^{u_{2}}
$$

with $c_{2}=c_{1} / d$. Since the right side of this inequality stays finite when $u \rightarrow-\infty$, we conclude that there is a constant $L>0$ with

$$
\frac{1}{L} \leq|f(u)| \leq L \quad \text { for all } u<u_{2} .
$$


Using that $\lambda$ depends smoothly on $\omega$ (see the Appendix), the constants $c_{1}, c_{2}, d$, and $L$ clearly can be chosen locally uniformly in $\omega$.

We substitute (3.15) into (3.14),

$$
\left|\frac{d}{d u} f\right| \leq c_{1} L e^{d u}
$$

This inequality shows that $f^{\prime}$ is integrable, and thus $f(u)$ converges for $u \rightarrow-\infty$. Setting

$$
f_{0}=\lim _{u \rightarrow-\infty} f(u) \stackrel{(3.15)}{\neq} 0
$$

we can integrate (3.16) from $-\infty$ to $u<b$ and get

$$
\left|f(u)-f_{0}\right| \leq c e^{d u}
$$

with $c=c_{1} L / d$. Substituting in the ansatz (3.8), we get (3.11).

¿From (3.9) we see that $X(u)$ does not decay to zero for $u \rightarrow-\infty$. As a consequence, the function $\Psi_{u_{2}}^{k \omega n}$ cannot have finite norm and thus is not a vector in the Hilbert space $\mathcal{H}_{u_{2}}$. This shows that the Hamiltonian $H_{u_{2}}$ has no point spectrum. In contrast to (3.3), we normalize the functions $\Psi_{u_{2}}^{k \omega n}$ according to

$$
\lim _{u \rightarrow-\infty}\left|X_{u_{2}}^{k \omega n}\right|=1, \quad\left(Y^{k \omega n} \mid Y^{k \omega n}\right)=1 .
$$

The next two lemmas describe the behavior of the normalization factors and the energy gaps as $u_{1} \rightarrow-\infty$.

Lemma 3.2. For fixed $u_{2}$ and asymptotically as $u_{1} \rightarrow-\infty$,

$$
\begin{aligned}
X_{u_{1}, u_{2}}^{k \omega n} & =\left.g\left(u_{1}\right) X_{u_{2}}^{k \omega n}\right|_{\left[u_{1}, u_{2}\right]} \quad \text { with } \\
\left|g\left(u_{1}\right)\right|^{-2} & =\left(u_{2}-u_{1}\right)+\mathcal{O}(1)
\end{aligned}
$$

Furthermore,

$$
\left|<\Psi_{u_{1}, u_{2}}^{k \omega n}\right| \Psi_{u_{1}, u_{2}}^{k \omega n^{\prime}}>-\delta^{n n^{\prime}}\left|\leq \frac{c}{u_{2}-u_{1}}<Y^{k \omega n}\right| \sin \vartheta \sigma^{1} \mid Y^{k \omega n^{\prime}}>,
$$

where the constant $c$ can be chosen locally uniformly in $\omega$.

Proof. Since $X_{u_{1}, u_{2}}^{k \omega n}$ and $X_{u_{2}}^{k \omega n}$ are solutions of the same ODE (3.13) with the same boundary conditions at $u_{2}$, they clearly coincide up to a normalization 
factor $g$, i.e. $X_{u_{1}, u_{2}}^{k \omega n}=g X_{u_{2}}^{k \omega n}$. Taking the norm on both sides and using the first part of (3.3), we obtain that

$$
\left|g\left(u_{1}\right)\right|^{-2}=\int_{u_{1}}^{u_{2}} \overline{X_{u_{2}}^{k \omega n}}(u) X_{u_{2}}^{k \omega n}(u) d u .
$$

We now substitute (3.8), multiply out, and use that $\left|f_{0}\right|^{2}=1$ according to the first part of (3.17), to obtain

$$
\left|g\left(u_{1}\right)\right|^{-2}=\int_{u_{1}}^{u_{2}}\left(1+\bar{X} R_{0}+\overline{R_{0}} X-\left|R_{0}\right|^{2}\right) d u .
$$

Since $X$ is bounded and $R_{0}$ has exponential decay (3.11), the last three summands in (3.21) are integrable, and thus $\left|g\left(u_{1}\right)\right|^{-2}-\left(u_{2}-u_{1}\right)$ is bounded uniformly in $u_{1}$. This proves (3.19).

The scalar product $<\Psi_{u_{1}, u_{2}}^{k \omega n} \mid \Psi_{u_{1}, u_{2}}^{k \omega n^{\prime}}>$ can be computed via (2.23). The orthonormality (3.4) yields that

$$
\begin{aligned}
& <\Psi_{u_{1}, u_{2}}^{k \omega n} \mid \Psi_{u_{1}, u_{2}}^{k \omega n^{\prime}}>-\delta^{n n^{\prime}} \\
& =a\left(X_{u_{1}, u_{2}}^{k \omega n}\left|\frac{\sqrt{\Delta}}{r^{2}+a^{2}} \sigma^{2}\right| X_{u_{1}, u_{2}}^{k \omega n^{\prime}}\right)\left(Y^{k \omega n}\left|\sin \vartheta \sigma^{1}\right| Y^{k \omega n^{\prime}}\right) .
\end{aligned}
$$

In order to estimate the radial scalar product, we first note that the factor $\sqrt{\Delta}$ goes exponentially to zero for $u \rightarrow-\infty$, and thus

$$
\left(X_{u_{1}, u_{2}}^{k \omega n}\left|\frac{\sqrt{\Delta}}{r^{2}+a^{2}} \sigma^{2}\right| X_{u_{1}, u_{2}}^{k \omega n^{\prime}}\right) \leq c_{4} \int_{u_{1}}^{u_{2}} e^{d u}\left|X_{u_{1}, u_{2}}^{k \omega n}\right|\left|X_{u_{1}, u_{2}}^{k \omega n^{\prime}}\right| d u
$$

for some constant $c_{4}>0$. Substituting (3.19) and using that the integral is uniformly bounded due to the factor $\exp d u$, we obtain the estimate

$$
\left|\left(X_{u_{1}, u_{2}}^{k \omega n}\left|\frac{\sqrt{\Delta}}{r^{2}+a^{2}} \sigma^{2}\right| X_{u_{1}, u_{2}}^{k \omega n^{\prime}}\right)\right| \leq c_{5}\left(u_{2}-u_{1}\right)^{-1},
$$

which together with (3.22) yields (3.20).

Lemma 3.3. The following estimate holds asymptotically as $u_{1} \rightarrow-\infty$,

$$
\Delta \omega_{k n}=\frac{\pi}{u_{2}-u_{1}}+\mathcal{O}\left(\left(u_{2}-u_{1}\right)^{-2}\right)
$$

for fixed $u_{2}$ locally uniformly in $\omega$. 
Proof. We consider solutions of (3.7) satisfying the boundary conditions at $u_{2}$ and ask for which values of $\omega$ and $\lambda_{n}(\omega)$ our boundary conditions are also fulfilled at $u_{1}$. As is immediately verified from (3.7),

$$
\frac{d}{d u}\left(\left|X_{+}\right|^{2}-\left|X_{-}\right|^{2}\right)=0 .
$$

Thus $\left|X_{+}\right|^{2}-\left|X_{-}\right|^{2}$ is independent of $u$, and since it vanishes at $u_{2}$,

$$
\left|X_{+}\right|^{2}=\left|X_{-}\right|^{2} \quad \text { for all } u \leq u_{2} .
$$

Hence for the boundary values at $u_{1}$, we need not be concerned about the absolute values of $X_{ \pm}$; it suffices to consider the condition for the phases

$$
\arg X_{+}\left(u_{1}\right)=\arg X_{-}\left(u_{1}\right) .
$$

It is convenient to work again with the ansatz (3.12). In order to describe the dependence on $\omega$, we differentiate (3.13) with respect to $\omega$. Since $\lambda$ depends smoothly on $\omega$, we obtain the bound

$$
\left|\frac{d}{d u} \partial_{\omega} f\right| \leq c_{1} e^{d u}\left|\partial_{\omega} f\right|+c_{3} e^{d u}|f|
$$

with constants $c_{1}, d$ as in (3.14) and $c_{3}>0$. Using that $|f|$ is bounded from above (3.15), we get

$$
\left|\frac{d}{d u}\left(\left|\partial_{\omega} f\right|+c_{4}\right)\right| \leq c_{1} e^{d u}\left(\left|\partial_{\omega} f\right|+c_{4}\right)
$$

with $c_{4}=c_{3} L / c_{1}$. Similar to the development after (3.14), dividing by $\left(\left|\partial_{\omega} f\right|+c_{4}\right)$ and integrating yields

$$
\left.\log \left(\left|\partial_{\omega} f\right|+c_{4}\right)\right|_{u} ^{u_{2}} \leq\left. c_{2} e^{d u}\right|_{u} ^{u_{2}}
$$

and since the right side of this inequality is uniformly bounded in $u$,

$$
\left|\partial_{\omega} f\right| \leq c_{5}
$$

for some constant $c_{5}>0$.

For the study of the phase shifts, we introduce the phase function

$$
\rho(u)=\arg f_{+}(u)-\arg f_{-}(u)-2 \Omega u_{2}
$$

(the last summand was included so that $\rho\left(u_{2}\right)=0$ ). The derivative of the argument of a complex-valued function $h$ is given by

$$
\frac{d}{d u} \arg h(u)=\operatorname{Im} \frac{h^{\prime}(u)}{h(u)} .
$$


Using this formula together with the fact that, according to (3.15) and (3.24) both $\left|f_{+}\right|$and $\left|f_{-}\right|$are bounded away from zero, we obtain that

$$
\left|\frac{d}{d u} \partial_{\omega} \rho\right| \leq 4 L\left|\frac{d}{d u} \partial_{\omega} f\right|+8 L^{2}\left|\partial_{\omega} f\right|\left|\frac{d}{d u} f\right| .
$$

Substituting in (3.26), (3.16) as well as the bounds (3.15) and (3.27), we conclude that

$$
\left|\frac{d}{d u} \partial_{\omega} \rho\right| \leq c_{6} e^{d u}
$$

with some constant $c_{6}>0$. We integrate this inequality from $u<u_{2}$ to $u_{2}$. Since $\rho\left(u_{2}\right)=0$ independently of $\omega$, the boundary term $\partial_{\omega} \rho\left(u_{2}\right)$ drops out, and we obtain the bound

$$
\left|\partial_{\omega} \rho(u)\right| \leq C \quad \text { for all } u \leq u_{2}
$$

with a constant $C>0$. This means that the equation for $f,(3.13)$ leads only to finite phase shifts.

The boundary conditions at $u_{1},(3.25)$, are fulfilled iff

$$
\Phi:=2 \Omega\left(u_{2}-u_{1}\right)+\rho=0(\bmod 2 \pi) .
$$

Differentiating with respect to $\omega$ and integrating again from $\omega_{I}$ to $\omega_{I I}, \omega_{I}<$ $\omega_{I I}$, we obtain that

$$
\begin{gathered}
\left|\Phi\left(\omega_{I I}\right)-\Phi\left(\omega_{I}\right)-2\left(\omega_{I I}-\omega_{I}\right)\left(u_{2}-u_{1}\right)\right| \\
\leq \int_{\omega_{I}}^{\omega_{I I}}\left|\partial_{\omega} \rho\right| d \omega \stackrel{(3.28)}{\leq} C\left(\omega_{I I}-\omega_{I}\right),
\end{gathered}
$$

and this proves $(3.23)$.

We can now prove the integral representation for the propagator of $H_{u_{2}}$.

Proposition 3.4. For every $\left.\Psi \in C_{0}^{\infty}\left(\left(-\infty, u_{2}\right]\right) \times S^{2}\right)^{4}$ and $x=(u, \vartheta, \varphi)$,

$$
\left(e^{-i t H_{u_{2}}} \Psi\right)(x)=\frac{1}{\pi} \sum_{k \in \mathbf{Z}} \int_{-\infty}^{\infty} d \omega e^{-i \omega t} \sum_{n \in \mathbf{Z}} \Psi_{u_{2}}^{k \omega n}(x)<\Psi_{u_{2}}^{k \omega n} \mid \Psi>
$$

Proof. According to the bound (3.20), the operator $A$ in (3.6) converges uniformly in $\omega$ and $k$ to the identity as $u_{1} \rightarrow-\infty$, and thus (3.5) simplifies asymptotically to

$$
\begin{aligned}
& \left(e^{-i t H_{u_{1}, u_{2}}} \Psi\right)(x) \\
& =\sum_{k \in \mathbf{Z}} \sum_{\omega \in \sigma\left(H_{u_{1}, u_{2}}^{k}\right)} e^{-i \omega t} \sum_{n \in N(k, \omega)} \Psi_{u_{1}, u_{2}}^{k \omega n}<\Psi_{u_{1}, u_{2}}^{k \omega n} \mid \Psi>+\mathcal{O}\left(\left(u_{2}-u_{1}\right)^{-1}\right) .
\end{aligned}
$$


Using (3.18) and (3.19), we can express $\Psi_{u_{1}, u_{2}}^{k \omega n}$ by $\Psi_{u_{2}}^{k \omega n}$,

$$
\begin{aligned}
& \left(e^{-i t H_{u_{1}, u_{2}}} \Psi\right)(x)=\sum_{k \in \mathbf{Z}} \frac{1}{u_{2}-u_{1}} \sum_{\omega \in \sigma\left(H_{u_{1}, u_{2}}\right)} e^{-i \omega t} \\
& \times \sum_{n \in N(k, \omega)} \Psi_{u_{1}}^{k \omega n}<\Psi_{u_{1}}^{k \omega n} \mid \Psi>_{u_{1}, u_{2}}+\mathcal{O}\left(\left(u_{2}-u_{1}\right)^{-1}\right) .
\end{aligned}
$$

The gap estimate, Lemma 3.3, shows that the sum over the spectrum is a Riemann sum which converges as $u_{1} \rightarrow-\infty$ to an integral.

The idea for proving an integral representation for $\exp (-i t H)$ is to take in (3.29) a suitable limit $u_{2} \rightarrow+\infty$. In preparation, we need to derive estimates which describe the asymptotics of solutions of the radial equation (3.7) for large $u$. In this regime,

$$
\begin{aligned}
\frac{d}{d u} X= & {\left[\left(\begin{array}{cc}
-i \omega & i m \\
-i m & i \omega
\end{array}\right)+\frac{1}{u}\left(\begin{array}{cc}
-i e Q & -i m M-\lambda \\
i m M-\lambda & i e Q
\end{array}\right)\right] X } \\
& +\mathcal{O}\left(u^{-2}\right) X
\end{aligned}
$$

Thus the matrix potential on the right converges for $u \rightarrow \infty$. If $|\omega|<m$, its eigenvalues $\lambda= \pm \sqrt{m^{2}-\omega^{2}}$ are real, and this leads to one fundamental solution of (3.30) which decays exponentially like $\exp \left(-\sqrt{m^{2}-\omega^{2}} u\right)$, and the other solution has exponential growth $\sim \exp \left(\sqrt{m^{2}-\omega^{2}} u\right)$. We denote these two fundamental solutions by $\Psi_{1}^{k \omega n}$ and $\Psi_{2}^{k \omega n}$, respectively, and normalize them according to

$$
\lim _{u \rightarrow-\infty}\left|\Psi_{1 / 2}^{k \omega n}(u)\right|=1
$$

where our notation 1/2 means that the above equation is valid in both cases 1 and 2. For $|\omega|>m$, on the other hand, the eigenvalues of the matrix potential at $u=\infty$ are imaginary, $\lambda= \pm i \sqrt{\omega^{2}-m^{2}}$, and this leads to two fundamental solutions $\Psi_{1 / 2}^{k \omega n}$ with oscillatory behavior $\sim \exp \left( \pm i \sqrt{\omega^{2}-m^{2}} u\right)$. For the normalization, we are now free to choose both the amplitude and the phase. Our convention is that

$$
f_{0,1}^{k \omega n}=\left(\begin{array}{l}
1 \\
0
\end{array}\right) \quad \text { and } \quad f_{0,2}^{k \omega n}=\left(\begin{array}{l}
0 \\
1
\end{array}\right)
$$

with $f_{0,1 / 2}^{k \omega n}$ as in the asymptotic expansion (3.8). The next lemma describes the asymptotics of the oscillatory solutions as $u \rightarrow \infty$. 
Lemma 3.5. Every nontrivial solution $X$ of (3.7) for $|\omega|>m$ has for large $u$ the asymptotic form

$$
X(u)=A\left(\begin{array}{cc}
e^{-i \Phi(u)} & f_{\infty}^{+} \\
e^{i \Phi(u)} & f_{\infty}^{-}
\end{array}\right)+R_{\infty}(u)
$$

with

$$
\begin{aligned}
f_{\infty} & \neq 0 \\
\Phi & =\epsilon(\omega)\left(\sqrt{\omega^{2}-m^{2}} u+\frac{\omega e Q+M m^{2}}{\sqrt{\omega^{2}-m^{2}}} \log u\right) \\
A & =\left(\begin{array}{cc}
\cosh \Theta & \sinh \Theta \\
\sinh \Theta & \cosh \Theta
\end{array}\right), \quad \Theta=\frac{1}{4} \log \left(\frac{\omega+m}{\omega-m}\right) \\
\left|R_{\infty}\right| & \leq \frac{C}{u}
\end{aligned}
$$

and a constant $C>0$.

Proof. We write (3.7) symbolically as

$$
X^{\prime}=V X
$$

with a matrix potential $V(u)$. According to (3.30) and the hypothesis $|\omega|>$ $m$, the eigenvalues of $V$ are, for sufficiently large $u$, purely imaginary. More precisely, there is a transformation matrix $B(u)$ with

$$
B^{-1} V B=-i \Omega \sigma^{3}
$$

and a suitable function $\Omega(u)$. Since the matrix potential $V$ converges for $u \rightarrow \infty$ and has a regular expansion in powers of $1 / u$, we can choose $B$ such that

$$
|B(u)| \leq c_{0}, \quad\left|B^{\prime}(u)\right| \leq \frac{c_{0}}{u^{2}}
$$

with a constant $c_{0}>0$. The transformed wave function $\left(B^{-1} X\right)$ satisfies the equation

$$
\frac{d}{d u}\left(B^{-1} X\right)=\left[-i \Omega(u) \sigma^{3}-B^{-1} B^{\prime}\right]\left(B^{-1} X\right)
$$

Hence employing the ansatz

$$
X=B\left(\begin{array}{c}
e^{-i \Phi} f^{+}(u) \\
e^{i \Phi} f^{-}(u)
\end{array}\right) \quad \text { with } \quad \Phi^{\prime}(u)=\Omega(u)
$$


and using the bound (3.39), we obtain the inequality

$$
\left|\frac{d}{d u} f\right| \leq \frac{c_{0}^{2}}{u^{2}}|f| .
$$

A short calculation shows that $\Phi$ has the explicit form (3.35), and that $B(u)=A+\mathcal{O}\left(\frac{1}{u}\right)$ with $A$ according to (3.36). The term of order $\mathcal{O}\left(\frac{1}{u}\right)$ can be absorbed into $R_{\infty}$.

The inequality (3.42) can be used similar to (3.14) in Lemma 3.1 Namely, dividing by $|f|$ and integrating yields for sufficiently large $u$ the bounds

$$
\frac{1}{L} \leq|f(u)| \leq L
$$

After substituting the upper bound for $|f|$ into (3.42), one sees that $f^{\prime}$ is integrable. Thus $f$ has a finite and, according to (3.43), non-zero limit,

$$
f_{\infty}:=\lim _{u \rightarrow \infty} f(u) \neq 0 .
$$

Finally, the $1 / u$-decay (3.37) follows by integrating (3.42) backwards from $u=\infty$ and employing the resulting bound in the ansatz (3.41).

In analogy to potential wall problems for Schrödinger operators, we call the function $f_{\infty}$ in (3.33) corresponding to our fundamental solutions $\Psi_{1 / 2}^{k \omega n}$ the transmission coefficients, and denote them by $f_{\infty 1 / 2}^{k \omega n}$.

Theorem 3.6. For every $\Psi \in C_{0}^{\infty}\left(\mathbb{R} \times S^{2}\right)^{4}$,

$$
\left(e^{-i t H} \Psi\right)(x)=\frac{1}{\pi} \sum_{k, n \in \mathbf{Z}} \int_{-\infty}^{\infty} d \omega e^{-i \omega t} \sum_{a, b=1}^{2} t_{a b}^{k \omega n} \Psi_{a}^{k \omega n}(x)<\Psi_{b}^{k \omega n} \mid \Psi>,
$$

where the coefficients $t_{a b}$ are for $|\omega|<m$ given by

$$
t_{a b}=\delta_{a, 1} \delta_{b, 1} .
$$

For $|\omega|>m$, the $t_{a b}$ are given by the integrals

$$
t_{a b}=\frac{1}{2 \pi} \int_{0}^{2 \pi} \frac{t_{a} \overline{t_{b}}}{\left|t_{1}\right|^{2}+\left|t_{2}\right|^{2}} d \alpha
$$

where the functions $t_{a}$ are related to the transmission coefficients by

$$
t_{1}(\alpha)=f_{\infty 2}^{+} e^{-i \alpha}-f_{\infty 2}^{-} e^{i \alpha}, \quad t_{2}(\alpha)=-f_{\infty 1}^{+} e^{-i \alpha}+f_{\infty 1}^{-} e^{i \alpha} .
$$

The integral and the series in (3.44) converge in norm in the Hilbert space $\mathcal{H}$. 
Proof. Our strategy is as follows. Choosing $u_{2}$ so large that supp $\Psi \subset$ $\left(-\infty, u_{2}\right)$, Proposition 3.4 yields for $t=0$ the "completeness relation"

$$
\Psi(x)=\frac{1}{\pi} \sum_{k \in \mathbf{Z}} \int_{-\infty}^{\infty} d \omega \sum_{n \in \mathbf{Z}} \Psi_{u_{2}}^{k \omega n}(x)<\Psi_{u_{2}}^{k \omega n} \mid \Psi>
$$

This formula remains true when $u_{2}$ is further increased,

$$
\Psi(x)=\frac{1}{\pi} \sum_{k \in \mathbf{Z}} \int_{-\infty}^{\infty} d \omega \sum_{n \in \mathbf{Z}} \Psi_{u_{2}+\tau}^{k \omega n}(x)<\Psi_{u_{2}+\tau}^{k \omega n} \mid \Psi>, \quad \tau \geq 0 .
$$

Hence we can take the average over $\tau$ in the finite interval $[0, T]$ with $T>0$ and obtain, using Fubini's theorem,

$$
\Psi=\frac{1}{\pi} \sum_{k \in \mathbf{Z}} \int_{-\infty}^{\infty} d \omega \sum_{n \in \mathbf{Z}}\left[\frac{1}{T} \int_{0}^{T} d \tau \Psi_{u_{2}+\tau}^{k \omega n}<\Psi_{u_{2}+\tau}^{k \omega n} \mid \Psi>\right] .
$$

We shall first prove that the square bracket in (3.49) has a finite limit as $T \rightarrow \infty$. Then we will show that for $T \rightarrow \infty$, we can in (3.49) take the limit inside the integral and the series in (3.49). This will give a decomposition of the identity in terms of eigensolutions of $H$, from which the representation of the propagator (3.44) will follow immediately by inserting the phase factors $\exp (-i \omega t)$.

Let us analyze the square bracket in (3.49). We can write $\Psi_{u_{2}+\tau}^{k \omega n}$ as a linear combination of the fundamental solutions $\Psi_{1 / 2}^{k \omega n}$,

$$
\Psi_{u_{2}+\tau}^{k \omega n}(x)=\sum_{a=1}^{2} c_{a}(\tau) \Psi_{a}^{k \omega n}(x),
$$

where the coefficients $c_{1 / 2}$ must be chosen such that our Dirichlet-type boundary conditions are satisfied at $u_{2}+\tau$. Then the square bracket becomes

$$
\frac{1}{T} \int_{0}^{T} d \tau \Psi_{u_{2}+\tau}^{k \omega n}<\Psi_{u_{2}+\tau}^{k \omega n}\left|\Psi>=\sum_{a, b=1}^{2} t_{a b}(T) \Psi_{a}^{k \omega n}<\Psi_{b}^{k \omega n}\right| \Psi>
$$

with

$$
t_{a b}(T)=\frac{1}{T} \int_{0}^{T} c_{a}(\tau) \overline{c_{b}(\tau)} d \tau
$$

In the case $|\omega|<m, \Psi_{1}^{k \omega n}$ and $\Psi_{2}^{k \omega n}$ are for large $u$ exponentially decaying and increasing, respectively. Hence in order to fulfill the boundary conditions at $u=u_{2}+\tau$, the quotient $c_{2}(\tau) / c_{1}(\tau)$ must go exponentially to zero. 
Moreover, our normalization conditions (3.17) and (3.31) imply that $\left|c_{1}(\tau)\right|^{2}$ must tend to one. We conclude that there is a constant $c_{1}$ with

$$
\left|c_{a}(\tau)-\delta_{a, 1}\right| \leq c_{1} e^{-\sqrt{m^{2}-\omega^{2}} \tau},
$$

and so (3.52) converges for $T \rightarrow \infty$ to (3.45). In the case $|\omega|>m$, the fundamental solutions are oscillating for large $u$, as described by Lemma 3.5. The boundary conditions at $u_{2},(2.21)$, imply that the following scalar product must vanish,

$$
<\left(\begin{array}{c}
f_{1}^{+} e^{-i \Phi\left(u_{2}+\tau\right)}-f_{1}^{-} e^{i \Phi\left(u_{2}+\tau\right)} \\
f_{2}^{+} e^{-i \Phi\left(u_{2}+\tau\right)}-f_{2}^{-} e^{i \Phi\left(u_{2}+\tau\right)}
\end{array}\right)+\mathcal{O}\left(\tau^{-1}\right),\left(\begin{array}{c}
c_{1}(\tau) \\
c_{2}(\tau)
\end{array}\right)>=0
$$

where $f_{1 / 2}$ are the transmission coefficients. Moreover, the normalization and phase conditions (3.17) and (3.32) yield that

$$
\left|c_{1}\right|^{2}+\left|c_{2}\right|^{2}=1 \text {. }
$$

The general solution to (3.53) is

$$
\left(\begin{array}{l}
c_{1} \\
c_{2}
\end{array}\right)=\frac{1}{D}\left(\begin{array}{c}
f_{2}^{+} e^{-i \Phi\left(u_{2}+\tau\right)}-f_{2}^{-} e^{i \Phi\left(u_{2}+\tau\right)} \\
-f_{1}^{+} e^{-i \Phi\left(u_{2}+\tau\right)}+f_{1}^{-} e^{i \Phi\left(u_{2}+\tau\right)}
\end{array}\right)+\mathcal{O}\left(\tau^{-1}\right)
$$

with a complex parameter $D$, which can be chosen so as to satisfy the normalization condition (3.54). We now substitute (3.55) into (3.52) and take $\Phi$ as the integration parameter,

$$
t_{a b}(T)=\frac{1}{T} \int_{\Phi(0)}^{\Phi(T)} c_{a}(\Phi) \overline{c_{b}(\Phi)} \frac{d \Phi}{\left|\Phi^{\prime}\right|} .
$$

Using (3.35), one sees that (3.56) converges for $T \rightarrow \infty$ to the average over one period, giving (3.46) and (3.47). We conclude that the bracket in (3.49) converges pointwise as $T \rightarrow \infty$.

Next we shall prove that in (3.49) we may take the limit $T \rightarrow \infty$ inside the series and the integral, and that for the resulting limit the series and the integral converge in norm. The sum over $k$ in (3.49) gives the decomposition into the eigenspaces of the angular operator $i \partial_{\varphi}$, which we denote by $\mathcal{H}^{k}$. We may consider the situation on each such eigenspace separately, and thus assume that $\Psi \in \mathcal{H}^{k}$. For the integral and the $n$-summation in (3.49) the situation is more difficult because the spectral decomposition of the Hamiltonian depends on $u_{2}$, and because the eigenvalues $\lambda_{n}$ of $\mathcal{A}$ and corresponding eigenspaces depend on $\omega$. We first apply to (3.48) the operator product $\mathcal{A}^{2 p} H^{2 q}$ with $p, q \geq 0$ (with $\mathcal{A}$ as in (2.4), where the $t$-derivative 
in $\mathcal{A}$ is carried out by applying the operator $-i H)$ and take the inner product with $\Psi$. This gives

$$
<\Psi\left|\mathcal{A}^{2 p} H^{2 q} \Psi>=\int_{-\infty}^{\infty} \frac{d \omega}{\pi} \omega^{2 q} \sum_{n \in \mathbf{Z}} \lambda_{n}^{2 p}(\omega)\right|<\Psi_{u_{2}+\tau}^{k \omega n}|\Psi>|^{2} .
$$

If we consider on the right side of (3.57) instead of $\left|\left\langle\Psi_{u_{2}+\tau}^{k \omega n} \mid \Psi\right\rangle\right|^{2}$ a mixed product with $\Phi, \Psi \in C_{0}^{\infty}\left(\left(-\infty, u_{2}\right) \times S^{2}\right)^{4} \cap \mathcal{H}^{k}$, we can in the integrand use the inequality $x y \leq \frac{1}{2}\left(x^{2}+y^{2}\right)$, and then apply (3.57) to obtain

$$
\begin{aligned}
& \sum_{n \in \mathbf{Z}} \int_{-\infty}^{\infty} \frac{d \omega}{\pi} \omega^{2 q} \lambda_{n}^{2 p}(\omega)|<\Phi| \Psi_{u_{2}+\tau}^{k \omega n}><\Psi_{u_{2}+\tau}^{k \omega n}|\Psi>| \\
& \quad \leq \frac{1}{2}\left(<\Phi\left|\mathcal{A}^{2 p} H^{2 q} \Phi>+<\Psi\right| \mathcal{A}^{2 p} H^{2 q} \Psi>\right)
\end{aligned}
$$

and this bound holds for all $\tau \geq 0$.

Let $\varepsilon>0$. Then for any $\omega_{0}>0,(3.58)$ yields for $p=0$ and $q=1$ that

$$
\begin{aligned}
& \sum_{n \in \mathbf{Z}} \int_{\mathbb{R} \backslash\left[-\omega_{0}, \omega_{0}\right]} \frac{d \omega}{\pi}|<\Phi| \Psi_{u_{2}+\tau}^{k \omega n}><\Psi_{u_{2}+\tau}^{k \omega n}|\Psi>| \\
& \quad \leq \frac{1}{\omega_{0}^{2}} \sum_{n \in \mathbf{Z}} \int_{-\infty}^{\infty} \frac{d \omega}{\pi} \omega^{2}|<\Phi| \Psi_{u_{2}+\tau}^{k \omega n}><\Psi_{u_{2}+\tau}^{k \omega n}|\Psi>| \\
& \quad \leq \frac{1}{2 \omega_{0}^{2}}\left(\|H \Phi\|^{2}+\|H \Psi\|^{2}\right) .
\end{aligned}
$$

We choose $\omega_{0}$ so large that

$$
\sum_{n \in \mathbf{Z}} \int_{\mathbb{R} \backslash\left[-\omega_{0}, \omega_{0}\right]} \frac{d \omega}{\pi}|<\Phi| \Psi_{u_{2}+\tau}^{k \omega n}><\Psi_{u_{2}+\tau}^{k \omega n}|\Psi>|<\frac{\varepsilon}{2}
$$

for all $\tau \geq 0$. This inequality allows us to restrict attention to $\omega$ in the finite interval $\left[-\omega_{0}, \omega_{0}\right]$. Next for a constant $n_{0}>0$, we consider $(3.58)$ for $p=1$ and $q=0$. This gives the inequality

$$
\begin{aligned}
& \sum_{|n|>n_{0}} \int_{-\omega_{0}}^{\omega_{0}} \frac{d \omega}{\pi}|<\Phi| \Psi_{u_{2}+\tau}^{k \omega n}><\Psi_{u_{2}+\tau}^{k \omega n}|\Psi>| \\
& \quad \leq \frac{1}{2}\left(<\Phi\left|\mathcal{A}^{2} \Phi>+<\Psi\right| \mathcal{A}^{2} \Psi>\right) \sup _{\omega \in\left[-\omega_{0}, \omega_{0}\right],|n|>n_{0}} \lambda_{n}^{-2}(\omega) .
\end{aligned}
$$

Clearly $\lambda_{n}^{2}(\omega) \rightarrow \infty$ for $n \rightarrow \pm \infty$ uniformly in $\omega \in\left[-\omega_{0}, \omega_{0}\right]$, and thus we can by choosing $n_{0}$ sufficiently large arrange that

$$
\sum_{|n|>n_{0}} \int_{-\omega_{0}}^{\omega_{0}} \frac{d \omega}{\pi}|<\Phi| \Psi_{u_{2}+\tau}^{k \omega n}><\Psi_{u_{2}+\tau}^{k \omega n}|\Psi>|<\frac{\varepsilon}{2} .
$$


Putting together the estimates (3.59) and (3.60), we conclude that

$$
\left(\sum_{n \in \mathbf{Z}} \int_{-\infty}^{\infty}-\sum_{|n|>n_{0}} \int_{-\omega_{0}}^{\omega_{0}}\right) \frac{d \omega}{\pi}|<\Phi| \Psi_{u_{2}+\tau}^{k \omega n}><\Psi_{u_{2}+\tau}^{k \omega n}|\Psi>|<\varepsilon
$$

for all $\tau \geq 0$.

For $\omega$ in a finite interval and $n$ in a finite set, we can take the average over $\tau \in[0, T]$ and take the limit $T \rightarrow \infty$ using Lebesgue's dominated convergence theorem (notice that according to Lemma 3.1, $\Psi_{u_{2}+\tau}^{k \omega n}$ is on the support of $\Phi$ and $\Psi$ uniformly bounded in $\tau$ ). This gives

$$
\begin{gathered}
\left.\lim _{T \rightarrow \infty} \sum_{n=-n_{0}}^{n_{0}} \int_{-\omega_{0}}^{\omega_{0}} \frac{d \omega}{\pi}\left[\frac{1}{T} \int_{0}^{T} d \tau<\Phi\left|\Psi_{u_{2}+\tau}^{k \omega n}><\Psi_{u_{2}+\tau}^{k \omega n}\right| \Psi\right\rangle\right] \\
=\sum_{n=-n_{0}}^{n_{0}} \int_{-\omega_{0}}^{\omega_{0}} \frac{d \omega}{\pi} \sum_{a, b=1}^{2} t_{a b}^{k \omega n}<\Phi\left|\Psi_{a}^{k \omega n}><\Psi_{b}^{k \omega n}\right| \Psi>
\end{gathered}
$$

with $t_{a b}$ according to (3.45) and (3.46). Since $\varepsilon$ in (3.61) can be chosen arbitrarily small and $n_{0} \rightarrow \infty, \omega_{0} \rightarrow \infty$ as $\varepsilon \searrow 0$, we obtain that (3.62) is true also for $n_{0}=\infty=\omega_{0}$, with absolute convergence of the integral and the series. Since $\Phi$ can be chosen arbitrarily, we conclude that

$$
\Psi=\lim _{T \rightarrow \infty}(3.49)=\sum_{k, n \in \mathbf{Z}} \int_{-\infty}^{\infty} \frac{d \omega}{\pi} \sum_{a, b=1}^{2} t_{a b}^{k \omega n} \Psi_{a}^{k \omega n}<\Psi_{b}^{k \omega n} \mid \Psi>
$$

The estimate (3.58) for $p=0=q$ remains true if we take the average over $\tau \in[0, \infty)$, and a homogeneity argument (as in the proof of the Schwarz inequality in Hilbert spaces) yields that

$$
\sum_{n \in \mathbf{Z}} \int_{-\infty}^{\infty} \frac{d \omega}{\pi}\left|\sum_{a, b=1}^{2} t_{a b}<\Phi\right| \Psi_{a}^{k \omega n}><\Psi_{b}^{k \omega n}|\Psi>| \leq\|\Phi\|\|\Psi\| .
$$

This bound shows that the integral and series in (3.63) converge in norm, and that $\Psi$ need not be an eigenvector of $i \partial_{\varphi}$. We finally apply the unitary operator $\exp (-i t H)$ on both sides of (3.63) to obtain (3.44).

Notice that the coefficients $t_{a b}$ given by (3.46) are bounded,

$$
\left|t_{a b}\right| \leq \frac{1}{2} \quad \text { for }|\omega|>m
$$


In the asymptotic region $u \rightarrow-\infty,(3.44)$ goes over to a Fourier representation in terms of the plane-wave solution (3.8). A careful analysis of this limiting case gives additional information on the coefficients $t_{a b}$, namely

$$
t_{11}=\frac{1}{2}=t_{22} \quad \text { for }|\omega|>m .
$$

However, the non-diagonal elements $t_{12}$ and $t_{21}$ remain undetermined. We shall not derive the relations (3.65) here, and will not use them in what follows.

\section{The Decay Estimates}

Using the integral representation for the propagator of the previous section, we can now prove the decay of the probabilities.

Proof of Theorem 1.1. According to the hypotheses of the theorem, the initial data $\Psi_{0}$ is in $L^{2}\left(\left(r_{1}, \infty\right) \times S^{2}, d \mu\right)^{4}$. Since the transformation of the

spinors $(2.1)$ is smooth and involves factors $\Delta^{\frac{1}{4}}$ and $\sqrt{r}$, we obtain for the transformed initial data $\hat{\Psi}_{0}$ that

$$
r^{-\frac{1}{2}} \Delta^{-\frac{1}{4}} \hat{\Psi}_{0} \in L^{2}\left(\left(r_{1}, \infty\right) \times S^{2}, d \mu\right)^{4} .
$$

Equivalently, computing the volume element on the hypersurface $t=$ const,

$$
\frac{r^{2}}{\Delta}\left|\hat{\Psi}_{0}\right|^{2} \in L^{1}\left(\left(r_{1}, \infty\right) \times S^{2}, d r d \cos \vartheta d \varphi\right)^{4}
$$

Transforming to the variable $u,(2.10)$, one sees that $\hat{\Psi}_{0}$ is in the Hilbert space with scalar product $(2.16), \hat{\Psi}_{0} \in \mathcal{H}$. For simplicity, we again omit the hat in what follows.

Let $\varepsilon>0$. Since the wave functions with compact support are dense in $\mathcal{H}$, then for this $\varepsilon$, there is a $\Psi_{I} \in C^{\infty}\left(\left(r_{1}, \infty\right) \times S^{2}\right)^{4}$ such that

$$
\left\|\Psi_{I}-\Psi_{0}\right\|<\varepsilon
$$

For the Cauchy problem with initial data $\Psi_{0}$, we have the integral representation of Theorem 3.6. Since the series in (3.44) converge in norm, we can choose $k_{0}$ and $n_{0}$ such that

$$
\left\|\Psi_{k_{0}, n_{0}}-\Psi_{I}\right\| \leq \varepsilon,
$$


where $\Psi_{k_{0}, n_{0}}$ is defined by

$$
\Psi_{k_{0}, n_{0}}(x)=\frac{1}{\pi} \sum_{k=-k_{0}}^{k_{0}} \sum_{n=-n_{0}}^{n_{0}} \int_{-\infty}^{\infty} d \omega \sum_{a, b=1}^{2} t_{a b}^{k \omega n} \Psi_{a}^{k \omega n}(x)<\Psi_{b}^{k \omega n} \mid \Psi_{I}>.
$$

Consider the integrand in (4.3) for fixed $k$ and $n$,

$$
\sum_{a, b=1}^{2} t_{a b}^{k \omega n} \Psi_{a}^{k \omega n}(x)<\Psi_{b}^{k \omega n} \mid \Psi_{I}>
$$

¿From (3.64) and the estimates of Lemma 3.1, one sees that (4.4) is bounded, locally uniformly in $x$ and $\omega$. Thus the norm convergence established in Theorem 3.6 implies that $(4.4)$ is in $L^{1}\left(\mathbb{R}, \mathbb{C}^{4}\right)$ as a function of $\omega$, with an $L^{1}$-bound locally uniform in $x$. Hence its Fourier transform is $L^{\infty}$ in $t$, locally uniformly in $x$. Furthermore, the Riemann-Lebesgue lemma [8] yields that its Fourier transform tends to zero as $t \rightarrow \infty$, pointwise in $x$. Since (4.3) involves only finitely many terms, we conclude that the solution of the Cauchy problem with initial data $\Psi_{k_{0}, n_{0}}$,

$\Psi_{k_{0}, n_{0}}(t, x)=\frac{1}{\pi} \sum_{k=-k_{0}}^{k_{0}} \sum_{n=-n_{0}}^{n_{0}} \int_{-\infty}^{\infty} d \omega e^{-i \omega t} \sum_{a, b=1}^{2} t_{a b}^{k \omega n} \Psi_{a}^{k \omega n}(x)<\Psi_{b}^{k \omega n} \mid \Psi_{I}>$,

is $L^{\infty}$ in $t$ locally uniformly in $x$, and $\lim _{t \rightarrow \infty} \Psi_{n_{0}, k_{0}}(t, x)=0$ for all $x$.

Choose $K_{\delta, R}$ as in the statement of the theorem. Since the metric and Dirac matrices in the probability integral (1.3) are smooth and bounded on the compact set $K_{\delta, R}$, the corresponding bilinear form is continuous on $\mathcal{H}$, i.e. there is a constant $c$ depending only on $\delta$ and $R$ such that for all $\Psi_{1}, \Psi_{2} \in \mathcal{H}$

$$
\int_{K_{\delta, R}}\left(\overline{\Psi_{1}} \gamma^{j} \Psi_{2}\right) \nu_{j} d \mu \leq c\left\|\Psi_{1}\right\|\left\|\Psi_{2}\right\|
$$

The solution to our original Cauchy problem is obtained by applying the unitary operator $\exp (-i t H)$ to $\Psi_{0}$,

$$
\begin{aligned}
\Psi(t) & =e^{-i t H} \Psi_{0} \\
& =e^{-i t H} \Psi_{k_{0}, n_{0}}+e^{-i t H}\left(\Psi_{I}-\Psi_{k_{0}, n_{0}}\right)+e^{-i t H}\left(\Psi_{0}-\Psi_{I}\right) \\
& =\Psi_{k_{0}, n_{0}}(t)+e^{-i t H}\left(\Psi_{I}-\Psi_{k_{0}, n_{0}}\right)+e^{-i t H}\left(\Psi_{0}-\Psi_{I}\right),
\end{aligned}
$$

where $\Psi_{k_{0}, n_{0}}(t)$ has the integral representation (4.5). We substitute this formula for $\Psi(t)$ into the probability integral, multiply out, and apply the 
estimate (4.6) as well as the unitarity of $\exp (-i t H)$ together with (4.1) and (4.2). This gives the inequality

$$
\begin{aligned}
& \int_{K_{\delta, R}}\left(\bar{\Psi} \gamma^{j} \Psi\right)(t, x) \nu_{j} d \mu \\
& \quad \leq \int_{K_{\delta, R}}\left(\overline{\Psi_{k_{0}, n_{0}}} \gamma^{j} \Psi_{k_{0}, n_{0}}\right)(t, x) \nu_{j} d \mu+4 c^{2} \varepsilon^{2}+4 c \varepsilon\|\Psi\| .
\end{aligned}
$$

We showed above that the integrand in the last integral is uniformly bounded and tends to zero pointwise as $t \rightarrow \infty$. Thus the integral converges to zero according to Lebesgue's dominated convergence theorem.

We remark that in the spherically symmetric case, the analytical method given above to prove that $\hat{\Psi}_{0} \in \mathcal{H}$ is an alternative to the nice geometric argument by Kay and Wald [7], who use the causal propagation property and a discrete symmetry of the maximally extended space-time at the bifurcation 2-sphere.

\section{A Nondegeneracy and Regularity of the Angular Eigenfunctions}

In this appendix, we shall consider the angular equations $(2.6),(2.8)$. As explained in [1, Appendix A], it is useful to write (2.6) as an eigenvalue equation in $\lambda$,

$$
\mathcal{A} Y=\lambda Y \quad \text { with } \quad \mathcal{A}=\left(\begin{array}{cc}
-a m \cos \vartheta & \mathcal{L}_{-} \\
-\mathcal{L}_{+} & a m \cos \vartheta
\end{array}\right) .
$$

Proposition A.1. For given $k$ and $\lambda \in \sigma(\mathcal{A})$, there is at most one eigensolution of (A.1), which we denote by $Y^{k}$, i.e.

$$
\mathcal{A} Y^{k}=\lambda Y^{k} \text {. }
$$

By continuously varying the parameter $\omega$, the eigenvalue equation (A.2) can be extended to all values of $\omega \in \mathbb{R}$. Both $\lambda$ and $Y^{k}$ depend smoothly on $\omega$.

Proof. The two fundamental solutions of (A.2) behave near $\vartheta=0$ like

$$
Y^{k}=\left(\vartheta^{k}+o\left(\vartheta^{k}\right), o\left(\vartheta^{k}\right)\right) \text { and } Y^{k}=\left(o\left(\vartheta^{-k-1}\right), \vartheta^{-k-1}+o\left(\vartheta^{-k-1}\right)\right),
$$

respectively. Depending on whether $k$ is $\geq 0$ or negative, the second or first fundamental solution diverges in the limit $\vartheta \rightarrow 0$. In $[1$, Appendix A] it was 
shown that the eigenfunctions $Y^{k}$ are bounded on $S^{2}$ and smooth except at the poles. Thus we can rule out one of the fundamental solutions and conclude that (A.2) has at most one solution.

Note that the solutions of (A.2) are the eigenvectors of $\mathcal{A}$ restricted to the eigenspace of the operator $i \partial_{\varphi}$ with eigenvalue $k$, which we denote by $\mathcal{H}^{k}$. Since the terms involving $\omega$ in (A.1) are a relatively compact perturbation, standard perturbation theory [6] yields that the spectrum of $\mathcal{A}_{\mid \mathcal{H}^{k}}$ depends continuously on $\omega$. As no degeneracies occur, each eigenvalue $\lambda$ gives rise to a unique continuous family of eigenvalues $\lambda(\omega)$. Standard perturbation theory without degeneracies [6] then yields that $\lambda(\omega)$ and the corresponding eigenvector $Y^{k}(\omega)$ depend smoothly on $\omega$.

Acknowledgments: We would like to thank McGill University, Montréal, and the Max Planck Institute for Mathematics in the Sciences, Leipzig, for support and hospitality. We are grateful to the referee for helpful suggestions.

The research of FK was supported by NSERC grant \# RGPIN 1054901998, of S in part by the NSF, Grant No. DMS-G-9802370, and of Y in part by the NSF, Grant No. 33-585-7510-2-30.

\section{References}

[1] F. Finster, N. Kamran, J. Smoller, and S.-T. Yau, "Non-existence of time-periodic solutions of the Dirac equation in an axisymmetric black hole geometry," gr-qc/9905047, Comm. Pure Appl. Math. 53 (2000) 902-929

[2] B. Carter, "Black hole equilibrium states," in Black holes/Les astres occlus, Ecole d' été Phys. Théor., Les Houches (1972)

[3] S. Chandrasekhar, "The solution of Dirac's equation in Kerr geometry," Proc. Roy. Soc. Lond. A 349, 571-575 (1976)

[4] D. Page, "Dirac equation around a charged, rotating black hole," Phys. Rev. D 14, 1509 (1976)

[5] N. Toop, "The thermal radiation of electrons from a charged spinning black hole in a cosmological background," preprint D.A.M.T.P., Cambridge (1976)

[6] T. Kato, "Perturbation Theory for Linear Operators," 2nd edition, Springer Verlag (1976) 
[7] B. Kay, R. Wald, "Linear stability of Schwarzschild under perturbations which are nonvanishing on the bifurcation 2-sphere," Classical Quantum Gravity 4 (1987) 893-898

[8] M. Reed, B. Simon, B., "Methods of Modern Mathematical Physics," Vol. II, Academic Press (1975) 